\title{
Thermodynamics of ion hydration and its interpretation in terms of a common model
}

\begin{abstract}
Yizhak Marcus
Department of Chemistry, University of California at Irvine, Irvine, CA 92717, USA, and Department of Inorganic \& Analytical Chemistry, The Hebrew University of Jerusalem, 91904 Jerusalem, Israel

Abstract - The relationship between the conventional standard molar thermodynamic quantities of hydration of an ion and the corresponding quantities of solvation, that are due entirely to its interactions with its aqueous environment, is presented. The TATB assumption, i.e., that quantities pertaining to the tetraphenylarsonium cation equal those pertaining to the tetraphenylborate anion, is applied to the standard enthalpy, entropy, and Gibbs energy of hydration of ions, and to the standard partial molar heat capacity and volume of aqueous ions. A model of the hydrated ion, consisting of a layer of completely immobilized water molecules surrounded by a dielectric continuum affected by the field of the ion and water the structure of which is modified, is presented. The thickness of the first layer and the number of water molecules in it is proportional to the radius of the ion. The model is shown to be compatible with all these thermodynamic quantities.
\end{abstract}

\section{INTRODUCTION}

The solvation process for any solute, according to Ben-Naim and Marcus (ref. 1 ), is its transfer from a fixed point in vacuum (an ideal gas phase) to a fixed point in the solution. This definition implies that the translational partition function of the solute particle is the same in the gas and the solution, or in other words, the entire volume of the solution is at its disposal, and not only a so called 'free volume'. This definition applies, of course, also to the hydration of ions, where it is customary to ignore the surface potential involved in such a transfer, or to apply the process simultaneously to ions of equivalent opposite charges. In the latter case it is necessary to divide the experimental thermodynamic quantities for the hydration among the ions, in order to arrive at quantities pertaining to the individual ions. Although the above definition applies to any concentration of the solute (including the neat solute) (ref. 1), we will limit ourselves here to a discussion of the standard thermodynamic quantities pertaining to the hydrated ions and to the process of hydration. In this way we consider only the state of infinite dilution of the solute in the solvent, where solute-solute interactions are absent. The thermodynamic quantities of hydration and the other properties of an aqueous ion pertain then solely to its interaction with the water in its environment (which may extend considerably away from the ion). Furthermore, strict additivity of the contributions of the individual ions, weighted by their stoichiometric coefficients, to the property of the whole electrolyte must be conformed to.

Conventionally, standard thermodynamic quantities of hydration or standard thermodynamic quantities pertaining to the aqueous ions involve the standard states of the hypothetical ideal gas at $P_{0}=1 \mathrm{~atm}(0.101325 \mathrm{MPa})$ pressure (or, more recently, at exactly $0.1 \mathrm{MPa}$, where applicable, and of the hypothetical ideal solution at the concentration of $1000 \mathrm{~mol} \mathrm{m^{-3 }}$ ( $1 \mathrm{~mol}^{-\mathrm{I}}$ ). For the Gibbs energy of hydration it is necessary to employ equal molar concentrations in the gas and the solution phases in order to conform to the above definition of solvation. Furthermore, in the enthalpy, heat capacity, and entropy (but not the Gibbs energy) of hydration terms in the isobaric expansibility of water appear. The thermodynamic quantities of hydration according to the present definition, marked by an *, as distinct from the conventional standard ones, marked by an , are related to the latter by the following expressions. 


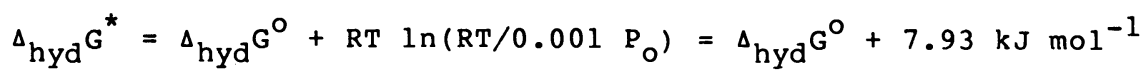

$$
\begin{aligned}
& \Delta_{h y d} H^{*}=\Delta_{h y d} H^{\circ}+R T(1-\alpha T)=\Delta_{h y d} H^{\circ}+2.29 \mathrm{~kJ} \mathrm{~mol}^{-1} \\
& \Delta_{h y d} S^{*}=\Delta_{h y d} S^{0}+R\left[1-\alpha T-\ln \left(R T / 0.001 P_{0}\right)\right]=\Delta_{h y d} S^{0}-18.9 \mathrm{~J} / \mathrm{K} \mathrm{mol}(3) \\
& \Delta_{h y d} C_{p}{ }^{*}=\Delta_{h y d} C_{p}{ }^{0}+R(1-2 \alpha T)=\Delta_{h y d} C_{p}{ }^{0}+7.0 \mathrm{~J} / \mathrm{K} \mathrm{mol} \\
& \Delta_{h y d} V^{*}=\Delta_{h y d} V^{0}-R T / P_{0}\left(-1+\kappa P_{o}\right)=\Delta_{h y d} V^{0}+24464 \mathrm{~cm}^{3} \mathrm{~mol}^{-1}
\end{aligned}
$$

Here $R$ is the gas constant, $T$ the absolute temperature, $\alpha$ the isobaric expansibility of water, and $k$ its isothermal compresssibility. Similar expressions hold for the thermal expansibility of hydration, the compressibility of hydration, etc. Since we deal here with just one solvent, water, we see that the additional terms are all constant at a given temperature and are independent of the solute.

The standard thermodynamic quantities of hydration of electrolytes are in many cases experimentally obtainable quantities. However, thermodynamics provides no clues to their proper division among the constituent ions of the electrolytes. Recourse to extrathermodynamic methods or assumptions is, therefore, necessary in order to make this division. This splitting can be applied to the thermodynamic quantity of hydration itself, symbolized by ${ }_{\text {hyd }} \mathrm{Y}^{\mathrm{O}}$, or to the two terms that make it up:

$$
\Delta_{h y d} Y^{\circ}=\Delta_{f} Y^{\circ}(a q)-\Delta_{f} Y^{\circ}(g) \text { or } \bar{Y}^{\infty}(a q)-Y^{\circ}(g)
$$

In the cases of the Gibbs energy and the enthalpy, $\Delta_{f} \mathrm{Y}^{\circ}$ designates the standard quantity of formation of the aqueous or the gaseous ion. in the cases of the entropy, the heat capacity and the volume, $\bar{Y}_{0}^{\infty}(a q)$ designates the standard partial molar quantity of the aqueous ion and $\mathrm{Y}^{\circ}(\mathrm{g})$ is the standard molar quantity of the gaseous ion. The individual ionic values of the standard molar entropies, heat capacities, and volumes are obtained from theory and, where required, from structural and spectroscopic data (ref. 2, $3)$. It is then only necessary to split the value of $\bar{Y}^{\infty}(a q)$ into the contributions from the individual ions.

The process of ion hydration, i.e., the transfer of the ion from the gas phase to water (at fixed positions) is similar to the process of ion transfer between two solvents (at fixed positions). The individual ionic thermodynamic functions of transfer of ions from a reference solvent (e.g., water) into some other solvent have been studied extensively by many authors and reviewed by Marcus (ref. 4, 5). Marcus has shown (ref. 6) that the least objectionable extrathermodynamic assumption for the splitting of the values of $\Delta+\mathrm{Y}^{\mathrm{O}}$ of electrolytes into the contributions from individual ions is the reference electrolyte method. The implementation of this method that has been tested and applied most extensively is the TATB one, which states that

$$
\Delta_{t r} \mathrm{Y}^{\circ}\left(\mathrm{Ph}_{4} \mathrm{As}^{+}\right)=\Delta_{t \mathrm{r}} \mathrm{Y}^{\circ}\left(\mathrm{BPh}_{4}{ }^{-}\right)=(1 / 2) \Delta_{t \mathrm{r}} \mathrm{Y}^{\circ}\left(\mathrm{Ph}_{4} \mathrm{AsBPh}_{4}\right)
$$

Here $Y$ represents the Gibbs energy, enthalpy, or entropy and $\mathrm{Ph}_{4} \mathrm{AsBPh}_{4}$ is tetraphenylarsonium tetraphenylborate (TATB) (ref. 6). The TATB assumption has also been applied to some other properties of aqueous ions, such as their standard partial molar heat capacities, studied by Abraham and Marcus (ref. 7) The applicability of the TATB assumption to the thermodynamics of hydration of ions and to the properties of aqueous ions has recently been discussed by Marcus (ref. 8 ).

If the TATB assumption is accepted as applying to the hydration of ions, then for any of the thermodynamic quantities dealt with in eqs. (1) to (5) the following should be valid:

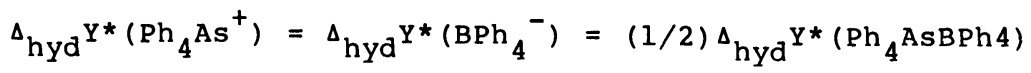

The additivity rule for solutes at infinite dilution then permits the use of data for electrolytes involving the $\mathrm{Ph}_{4} \mathrm{As}^{+}$cation and the $\mathrm{BPh}_{4}$ anion with the counter-ions $\mathrm{A}$ and $\mathrm{C}^{+}$, respectively, and data for the electrolyte CA to 
yield the individual ionic values. For example:

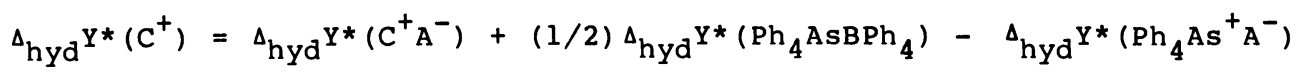

Once valid values for individual ions are available, they may be compared to suitable models for the ion in its aqueous environment. The best model should be able to accomodate all the different thermodynamic quantities pertaining to the hydration of the ion and to the properties of the aqueous ion.

\section{THE MODEL}

The model that appears to meet the requirements is similar in many respects to models that have been presented previously by others for explaining some of the thermodynamic data discussed here. It resembles most the model used by Abraham and Liszi (ref. 9), in that it involves the cavity in which the ion is situated in the water, a completely immobilized first hydration shell, and then surroundings, in which on the one hand the structure of the water is modified and on the other the water behaves as a dielectric continuum that is affected by the field of the ion (see Fig. 1). To summarize, application of this model specifies the thermodynamic functions of hydration of an ion of radius $r$ and charge $z$ to be

$$
\Delta_{h y d} \mathrm{Y}^{*}=\Delta \mathrm{Y}_{\mathrm{Nt}}(\mathrm{r})+\Delta \mathrm{Y}_{\mathrm{Ell}}(\mathrm{r}, \mathrm{z})+\Delta \mathrm{Y}_{\mathrm{El} 2}(\mathrm{r}, \mathrm{z})+\Delta \mathrm{Y}_{\mathrm{St}}(\mathrm{r}, \mathrm{z})
$$

where the terms on the right hand side pertain to the contributions from the neutral solute term, the term describing the immobilized solvent in the first layer around the ion, the Born term for the electrostatic effects beyond this first layer, and the term describing the effect on the structure of the water, respectively.

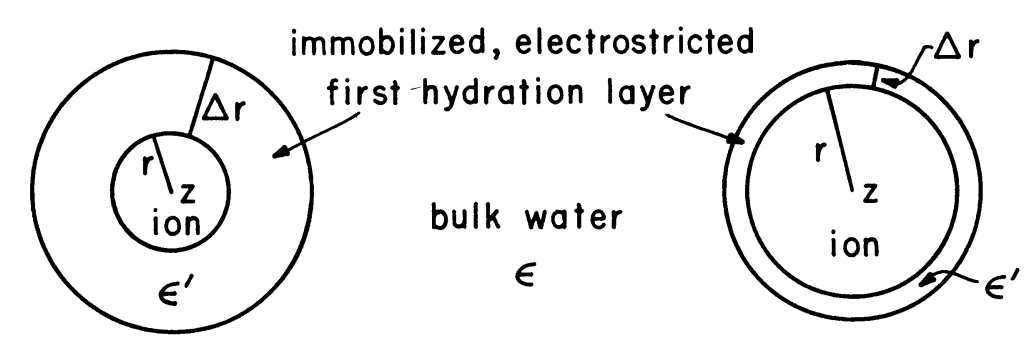

Fig. 1. Schematic representations of hydrated ions with high (left) and low (right) electrostatic fields, $|z| / r$.

The consequences of this model for the thermodynamics of hydration and for the thermodynamic properties of the hydrated ions can be evaluated from certain generally applying fitting constants and independent data, except for the modification of the structure of the water beyond the first immobilized hydration shell. However, the qualitative predictions of whether the structure of the water is enhanced or reduced and the extent of this modification can also be learned from the model.

The transfer of the ion from a fixed position in the gas phase to a fixed position in water is considered as proceeding in three stages. In the first the ion is discharged, in the second it is transferred as a neutral species having the same size as the (bare) ion in the solution, and in the third it is recharged up to its original charge. The electrostatic work in the gas phase is ignored (ref. 10), since it cancels for equivalent amounts of cations and anions. The work that is done when the (neutral) ion is transferred consists of the endoergic work required to produce the cavity that contains it in the solution and the mainly exoergic results of its interaction with its surroundings, once placed in this cavity. The latter arises from the dispersion forces and dipole-induced dipole forces that a neutral, polarizable solute would undergo. This work, as well as its temperature and pressure coefficients, are therefore evaluated from the corresponding experimental data on the thermodynamics of solution of noble gas and other suitable gaseous solutes in water: $\Delta \mathrm{Y}_{\mathrm{Nt}}(\mathrm{r})$. 
A key quantity in this, and subsequently developed, aspects of the model is the size of the ion in the aqueous solution at infinite dilution. Marcus (ref. 1l) has shown that both experimental evidence (from x-ray and neutron diffraction, and now also from ExAFs measurements) and computer simulation data (by Monte Carlo and molecular dynamics methods) support the use of the pauling crystal ionic radii for the radii of monoatomic ions in aqueous solutions. The small amount of experimental data available on polyatomic ions support the use of thermochemical or similar radii for the same purpose. Therefore the cavity term is evaluated from the solution thermodynamics of gaseous solutes having radi equal to the $r$ values of the ions in solution.

Once the (neutral) ion is in its cavity and interacts with its surroundings, the charge of the ion is turned back 'on', with a large electrostatic effect (except for the largest ions with single charges). This effect is equivalent to complete dielectric saturation and to the immobilization of the water molecules in a layer of solvent around the (bare) ion. The number $n$ of water molecules in this layer is proportional to the charge and the reciprocal of the radius of the ion (ref. 12); its thickness $\Delta r$ is calculated as shown below. A step function is imposed on the relative permittivity that varies smoothly with the distance from the center of the ion, but it is chosen so as to represent this function closely enough. This treatment yields the term $\Delta \mathrm{Y}_{\mathrm{E} 11}(\mathrm{r}, \mathrm{z})$.

Beyond this layer the ion still interacts electrostatically with the water in its surroundings, and the Born equation is applied to this region (ref. 10), with the bulk values of the relative permittivity of water and its temperature and pressure coefficients. The parameter of this term, $\Delta Y_{E / 2}(r, z)$, is $r$ $+\Delta r$, the sum of the radius of the ion plus the thickness of the first layer.

Finally the model recognizes the effect of the ion together with its first layer of translationally immobilized water molecules on the structure of the water in its environment. The hydrogen bonding of water molecules to each other is affected by the presence of an entity that is capable of accepting hydrogen bonds (in the case of anions with their first layers) or of donating them (in the analogous case of cations). The central charge has also an effect, but this is taken into account by the Born term. At the present stage of its development the model is incapable of a direct evaluation of this effect on the structure of water, and $\Delta Y S_{S t}(r, z)$ must be evaluated indirectly by difference. A constraint on this evaluation is imposed by the experimental data on the structural effects available from non-thermodynamic methods, such as nmr or viscosity measurements.

For the Gibbs energy of hydration the second and third terms in eq. (10) are:

$\Delta G_{E 11}+\Delta G_{E 12}=-\left(N_{A v} e^{2} / 8 \pi \varepsilon_{0}\right) z^{2}\left[\left(1-1 / \varepsilon^{\prime}\right)(\Delta r / r(r+\Delta r))+(1-1 / \varepsilon) /(r+\Delta r)\right]$

where $\varepsilon$ is the relative permittivity of bulk water and $\varepsilon^{\prime}$ is that of the dielectrically saturated first layer. For the other thermodynamic functions of hydration the appropriate pressure and temperature derivatives are taken, only $\varepsilon$ and $\varepsilon^{\prime}$ depending on these variables, the dependence of $\varepsilon$ on them being known from experimental data. The values of $\varepsilon^{\prime}$ and its dependence on the temperature and pressure were specified by setting $\varepsilon^{\prime}=n^{2}$ ' the square of the refractive index of water at the $D$ line, and taking the appropriate derivatives of this experimental value. Abraham et al. (ref. 13) chose somewhat different values.

The value of $\Delta r$, the thickness of the first layer, was taken to depend on $r$ in the following manner. The volume of this layer, $(4 \pi / 3)\left[(r+\Delta r)^{3}-r^{3}\right]$, is occupied by $n$ water molecules of volume $\pi \mathrm{d}^{3} / 6$ each, where $d=0.276 \mathrm{~nm}$ is their diameter. Their number is given by $n=A|z| / r$ (ref. 12), i.e.', is proportional to the charge and to the reciprocal of the radius of the ion, the proportionality factor A being a fitting parameter, the same for all ions. $A=0.36 \mathrm{~nm}$ serves well, but $\Delta \mathrm{r}$ depends only on its cube root. For singly charged ions this thickness is $\Delta r=0.19,0.07$, and 0.026 nm for ions of $r=0.06,0.14$ and $0.22 \mathrm{~nm}$ (corresponding roughly to $\mathrm{Li}^{+}, \mathrm{K}^{+}, \mathrm{and}^{-}$) respectively, and for singly, doubly and triply charged ions of $r=0.10 \mathrm{~nm}$ (corresponding roughly to $\mathrm{Na}^{+}, \mathrm{Ca}^{2+}$ and $\mathrm{Pr}^{3+}$ ) $\Delta \mathrm{r}=0.12,0.17$ and $0.21 \mathrm{~nm}$, respectively. Abraham et al. used for this thickness either one half of the diameter of the solvent molecule $(0.14$ or $0.15 \mathrm{~nm}$ for water, refs. 7,9$)$ or 
let it vary as a fitting parameter for $\Delta \mathrm{Ag}^{\circ}$ at $298 \mathrm{~K}$ (ref. 13). Latimer et al. (ref. 14) implicitly specified $\varepsilon^{\prime}=1$, hyd independent of the temperature, and $\Delta r=0.085 \mathrm{~nm}$ for alkali metal cations and $0.025 \mathrm{~nm}$ for halide anions in their fits of $\Delta_{h y d} H^{\circ}$ and $\Delta_{h y d} G^{\circ}$ at $298 \mathrm{~K}$.

Since $\Delta Y_{N t}$ is obtained from (interpolated) experimental data on a neutral gaseous Solute having the same radius $r$ as the ion, and $\Delta G_{E_{11}}+\Delta G_{E_{12}}$ are calculated by eq. (11), it remains to specify the values of the factor $A$ and of the term $\Delta \mathrm{Y}_{S t}$ in order to fit the thermodynamic functions of hydration. It should be noted that since structured and unstructured water around the ion are at equilibrium, $\Delta_{\text {st }} G=0$ for all ions. Also, the evidence points to the sodium and to the tetraethylammonium ions as being without noticeable effect on the structure of water (ref. 12). At least the signs of the contributions of $\Delta Y_{\text {St }}$ to the enthalpy and entropy of hydration can thus be estimated from fitting the experimental data of the individual ions, obtained, in turn, by the application of the TATB assumption, as explained above.

\section{ENTHALPY OF HYDRATION OF IONS AT 298.15 K}

The enthalpy of hydration of an ion is obtained from eq. (2) and the standard molar enthalpies of formation of the aqueous and the gaseous ion, eq. $(6)$ :

$$
\Delta_{h y d}{ }^{*}(\text { ion })=\Delta_{f} H^{\circ}(\text { ion, aq })-\Delta_{f} H^{\circ}(\text { ion,g })+2.29 \mathrm{~kJ} \mathrm{~mol}^{-1}
$$

Standard enthalpies of formation of the aqueous ions are tabulated in the NBS tables (ref. 15) on the conventional basis that $\Delta_{f} \mathrm{H}^{\circ}\left(\mathrm{H}^{+}, \mathrm{aq}\right)=0$ at all temperatures. Standard enthalpies of formation of the gaseous ions are obtained from lattice enthalpies of suitable crystalline compounds and their standard enthalpies of formation.

Following Marcus (ref. 16), the TATB assumption is applied to obtain the enthalpies of hydration by means of the thermodynamic cycle:

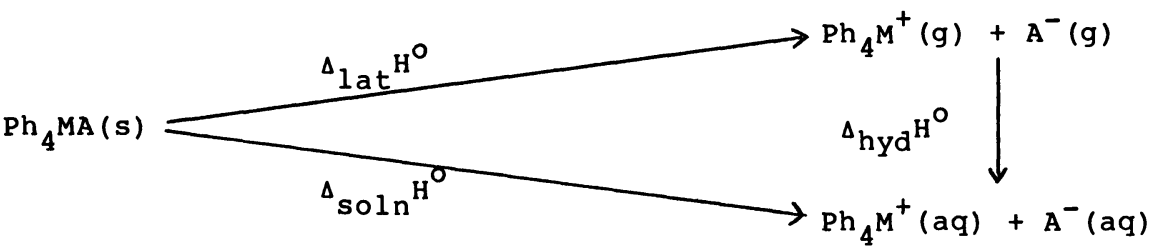

and a similar cycle for a salt involving a cation $\mathrm{C}^{+}$and the anion $\mathrm{BPh}_{4}^{-}$. The standard enthalpies of solution are known and the lattice enthalpies can be estimated, hence the standard enthalpies of hydration of the two tetraphenyl ion salts are known. The standard enthalpy of hydration of the salt CA is the difference between the sum of the conventional standard enthalpies of formation of the aqueous ions $\mathrm{C}^{+}$and $\mathrm{A}^{-}$and the sum of the standard enthalpies of formation of these gaseous ions. In the former of these sums the convention is no longer relevant. The standard enthalpy of hydration of TATB is then evaluated from the additivity condition:

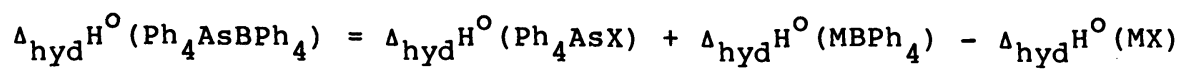

The TATB assumption is applied by splitting the left hand side of eq. (14) into two halves, to give the standard molar enthalpy of hydration of each reference ion of $-47 \mathrm{~kJ} \mathrm{~mol}^{-1}$. The standard molar enthalpies of hydration of the counter ions $\mathrm{C}^{+}$and $\mathrm{A}^{-}$, and indeed of any other ion, can then be obtained by use of the additivity rule, eq. (9). Specifically, $\Delta_{\text {a }} \mathrm{H}^{\circ}\left(\mathrm{H}^{+}\right)=-1103 \pm 7 \mathrm{~kJ}$ mol-1 results from this treatment, in good agreement whth the 'best' value suggested by Conway in his review of single ion thermodynamic quantities (ref. 17).

The model can now be applied to these single ion standard enthalpies of hydration with eqs. (10) and (2). The terms used in the former are $\Delta \mathrm{H}_{\mathrm{Nt}}=35$ - $267\left(\mathrm{r} / \mathrm{nm}\right.$ ) $\mathrm{kJ} \mathrm{mol}^{-1}$ (from data in ref. 13), $\Delta \mathrm{H}+\Delta \mathrm{H}_{1}=-69.5 \mathrm{z} \times$ $[0.35(\Delta \mathrm{r} / \mathrm{r})+1.005] /(\mathrm{r}+\Delta \mathrm{r}) \mathrm{kJ} \mathrm{mol}^{-1}$, from thellvalues aland temperature derivatives of $\mathrm{n}_{\mathrm{p}}$ and $\varepsilon$ of water, and an unknown $\Delta \mathrm{H}_{\text {st' }}$ which, however, must equal $T \Delta S_{s t}$, since $\Delta G_{S t}=0$, as explained above. However, except for the univalent ions, $\Delta \mathrm{H}_{\mathrm{s}}$ istmuch smaller than the sum of the electrostatic terms, which completely doninate $\Delta_{H^{*}}$ for the multivalent ions. Fig. 2 shows that the model does indeed fit theddata well. 


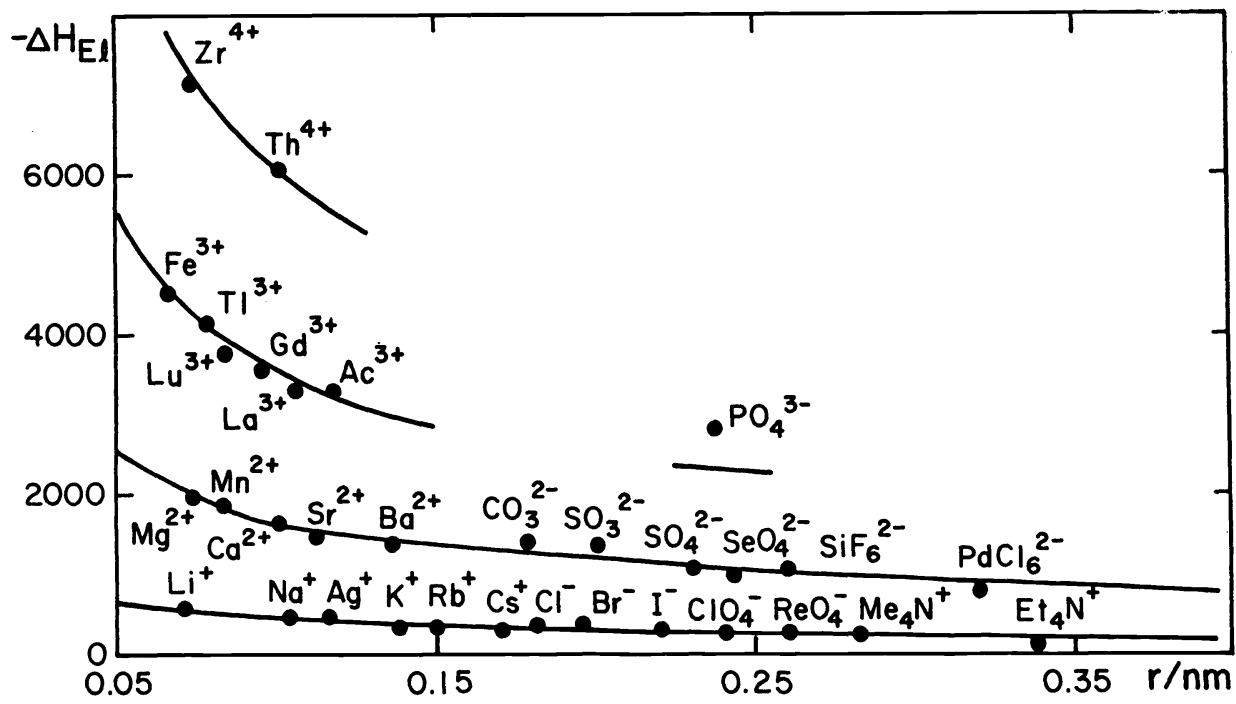

Fig. 2. The electrostatic contribution to the standard molar enthalpy of hydration of ions (lines) and the observed values on the TATB assumption (circles).

\section{ENTROPY OF HYDRATION OF IONS AT 298.15 K}

Entropies, contrary to enthalpies and Gibbs energies, can be put on an absolute scale by means of the third law of thermodynamics and statistical calculations. Cycles similar to eq. (13) can be written, where the quantities are standard molar entropies or standard partial molar entropies:

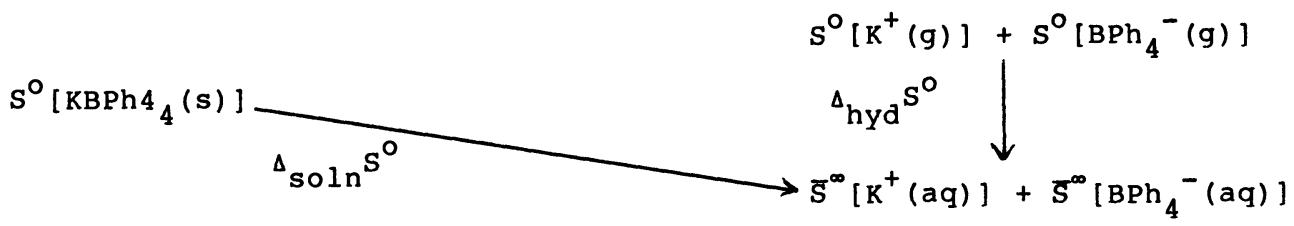

The standard molar entropies of the gaseous tetraphenyl-ions have been calculated from their structural and spectroscopic data by Marcus and Loewenschuss (ref. 18) and those of the monoatomic alkali metal or halide ions are available in the NBS tables (ref. 15). Standard entropies of solution of several tetraphenyl-salts are also available, so that the sum of the standard partial molar entropies of their constituent ions can be evaluated, provided the standard molar entropies of the crystalline salts are known. The latter quantity is, in fact, known for potassium tetraphenylborate from the work of Davies and stavely (ref. 19), but not for any salt of tetraphenylarsonium or -phosphonium. Pending its determination for a suitable salt of these cations, the TATB assumption cannot be applied in the same manner as for the enthalpy of hydration in the previous section.

There are no tabulated data of standard partial molar entropies of aqueous salts of the tetraphenyl-ions, or, what is the same thing, of the corresponding conventional quantities of these ions. Therefore the TATB assumption cannot be applied to obtain from them individual ionic standard partial molar entropies of aqueous ions. On the other hand, the TATB assumption was applied by Cox and Parker (ref. 20) to the standard molar entropies of solution of TATB in water, yielding the value $\Delta$ s $\mathrm{s}^{\circ}=-56 \mathrm{~J} \mathrm{~K} \mathrm{~K}^{-1}$ mol-1 for each of the reference ions. An estimate of the partial molar entropy of the tetraphenylborate anion of $346 \mathrm{~J} \mathrm{~K}^{-1} \mathrm{~mol}^{-1}$ was made by Marcus and Loewenschuss (ref. 18) on this basis. The TATB assumption would then hold this value to apply also to the tetraphenylarsonium (and -phosphonium) cation. This value, together wifh the standard molar entropy of the gaseous tetraphenylborate ion of $656 \mathrm{~J}$ $\mathrm{K}^{-1}$ mol-1, yields the standard molar entropy of hydration of this ion, $\Delta{ }^{-1} \mathrm{~s}^{\circ}$ $=-310 \pm 6 \mathrm{~J} \mathrm{~K} \mathrm{~K}^{-1}$ mol-1. Values of $\Delta$ d of other ions can then be calculated by means of the additivity rule, e.g., eq. (9). 
Application of eqs. (10) and (3) tests our model against these values. Contrary to the case of the enthalpies of hydration, in the entropies of hydration the neutral term, $\Delta S_{\mathrm{Nt}}=-22-600(\mathrm{r} / \mathrm{nm}$ ) J/K mol (from data in ref. 13), becomes of prime importance, especially for the larger univalent ions. On the other hand, the two electrostatic terms, $\Delta S_{E l l}+\Delta S_{E l 2}=4.06 z^{2} \times$ $[1.48(\Delta r / r)+1] /(r+\Delta r)$, play a less important role. The sum of these three terms ${ }_{*}$ is sometimes more positive, sometimes more negative than the values of ${ }_{\text {hyd }}{ }^{*}$ for the univalent ions, but for most multivalent ones it is more positive. This shows the necessity of including a negative water-structureaffecting term for the structure-making strong-field ions. The value of $\Delta S_{\text {St }}$ obtained by difference, comes out to be near zero for the sodium athd tetraethylammonium ions, as mentioned above, and to be positive for the known structure-breaking ions, such as cesium, bromide, or perchlorate. Altogether, the results of this treatment, Table 1 , agree well with those from a previous attempt to understand the entropies of hydration (ref. 12).

TABLE 1. The size (in $\mathrm{nm}$ ), number of entirely immobilized water molecules, thickness of electrostricted water layer (in $\mathrm{nm}$ ), and the water-structure-effect contributions to the standard molar entrppy and heat capacity (in $\mathrm{J} \mathrm{K}^{-1} \mathrm{~mol}^{-1}$ ) and volume (in $\mathrm{cm}^{3}$ mol ${ }^{-1}$ ) of selected ions.

\begin{tabular}{|c|c|c|c|c|c|c|}
\hline Ion & $\mathbf{r}$ & $\Delta r$ & $\mathrm{n}$ & $\Delta S_{S t}$ & $\Delta C_{p s t}+140$ & $\Delta \mathrm{v}_{\mathrm{St}}$ \\
\hline $\mathrm{Li}^{+}$ & 0.069 & 0.171 & 5 & -40 & 270 & 10 \\
\hline $\mathrm{Na}^{+}$ & 0.102 & 0.116 & 3 & 0 & 120 & 0 \\
\hline $\mathrm{K}^{+}$ & 0.138 & 0.075 & 3 & 30 & 0 & 0 \\
\hline $\mathrm{Cs}^{+}$ & 0.170 & 0.050 & 2 & 80 & -100 & 0 \\
\hline $\mathrm{Br}^{-}$ & 0.196 & 0.037 & 2 & 90 & -110 & 0 \\
\hline $\mathrm{I}^{-}$ & 0.220 & 0.028 & 2 & 130 & -140 & 0 \\
\hline $\mathrm{ClO}_{4}^{-}$ & 0.240 & 0.023 & 1 & 120 & -120 & 0 \\
\hline $\mathrm{Me}_{4} \mathrm{~N}^{+}$ & 0.280 & 0.016 & 1 & 50 & -100 & 30 \\
\hline $\mathrm{Et}_{4} \mathrm{~N}^{+}$ & 0.337 & 0.010 & 1 & 0 & 0 & 50 \\
\hline $\mathrm{Mg}^{2+}$ & 0.072 & 0.225 & 10 & -100 & 680 & 30 \\
\hline $\mathrm{Ca}^{2+}$ & 0.100 & 0.169 & 7 & -40 & 370 & 10 \\
\hline $\mathrm{Ba}^{2+}$ & 0.136 & 0.118 & 5 & -10 & 120 & 0 \\
\hline $\mathrm{CO}_{3}{ }^{2-}$ & 0.178 & 0.076 & 4 & -40 & -40 & -10 \\
\hline $\mathrm{SO}_{4}^{2-}$ & 0.230 & 0.045 & 3 & 20 & -180 & -20 \\
\hline $\mathrm{PdCl}_{6}{ }^{2-}$ & 0.32 & 0.02 & 2 & 160 & & \\
\hline $\mathrm{Sc}^{3+}$ & 0.075 & 0.260 & 14 & -30 & & \\
\hline $\mathrm{La}^{3+}$ & 0.105 & 0.197 & 10 & -40 & 770 & 20 \\
\hline $\mathrm{PO}_{4}^{3-}$ & 0.238 & 0.057 & 4 & -120 & -180 & \\
\hline $\operatorname{Th}^{4+}$ & 0.100 & 0.236 & 14 & -90 & & 80 \\
\hline $\mathrm{Fe}(\mathrm{CN})_{6}^{4-}$ & 0.44 & 0.02 & 3 & 140 & -570 & \\
\hline
\end{tabular}

\section{GIBBS ENERGY OF HYDRATION AT 298.15 K}

The Gibbs energy of hydration of an ion can be obtained from eqs. (1) and (6), i.e., from the difference in the standard molar Gibbs energy of formation of the aqueous and of the gaseous ion. The latter piece of information is generally not available directly, but can be obtaineg via the standard molar enthalpy of formation of the gaseous ion, $\Delta_{f}{ }^{O}(g)$, its 
standard molar entropy, $S^{\circ}(g)$, and the standard molar entropies of the elements and extra electrons then constitute the ion. Alternatively, the Gibbs energy of hydration can be obtained as the enthalpy of hydration minus the absolute temperature times the entropy of hydration, and this seems to be the better way.

The combination of $\Delta_{\text {hyd }} \mathrm{H}^{\circ}\left(\mathrm{MPh}_{4}^{ \pm}\right)=-47 \pm 5 \mathrm{~kJ} \mathrm{~mol}{ }^{-1}$ and $\Delta_{\text {hyd }} \mathrm{S}^{\circ}\left(\mathrm{MPh}_{4}^{ \pm}\right)=-310 \pm 6$ $\mathrm{J} \mathrm{K}^{-1} \mathrm{~mol}^{-1}$ yields for each of the reference ions the standard Gibbs energy of hydration $\Delta_{\text {hyd }} \mathrm{G}^{\circ}\left(\mathrm{MPh}_{4}^{ \pm}\right)=45 \pm 6 \mathrm{~kJ}$ mol-1. This positive value signifies that the work required for the creation of the big cavity in water into which the reference ion is to be transferred from the gas phase is larger than the Gibbs energy released by the dispersion and ion-multipole interactions such a large ion undergoes with its aqueous environment, once placed in the cavity. The standard Gibbs energies of hydration of other ions are then obtained, via eq. (9) or a similar one for anions, for all the other ions, for which both standard enthalpy and entropy of hydration data exist.

Application of the model to these data, by means of eq. (10), shows the dominance of the electrostatic interactions, as for the enthalpy of hydration. The neutral term is $\Delta G_{\text {t }}=41-87(\mathrm{r} / \mathrm{nm}) \mathrm{kJ} \mathrm{mol}^{-1}$ (from the data in ref. 13), the electrostatic termStare $\Delta G_{E \|}+\Delta G_{E_{12}}=-64.5 \mathrm{z}^{2}[0.44(\Delta \mathrm{r} / \mathrm{r})+0.987] /(\mathrm{r}$ $+\Delta r)$, and the structural term is zero. The general picture that emerges is not very different from Fig. 2 .

\section{HEAT CAPACITY OF HYDRATION OF IONS AT 298.15 K}

Contrary to the situation concerning the partial molar entropies of aqueous salts of the tetraphenyl-ions at infinite dilution, there are data in the literature on the corresponding heat capacities at constant pressure. Values of $C_{p}{ }^{\infty}(\mathrm{aq})$ of tetraphenylarsonium chloride, tetraphenylphosphonium chloride and bromide, and sodium tetraphenylborate (ref. 7, 21) can be combined with those of sodium chloride and bromide according to an equation analogous to eq. (14) to provide three independent estimates of $\bar{C}_{p}^{\infty}(\mathrm{aq})$ of the reference ions. The two values originating from the data for the phosphonium $\mathbf{s}_{\infty}$ ths are mutually consistent, yielding $\overline{\mathrm{C}}_{\mathrm{p}}^{\infty}\left(\mathrm{Ph}_{4} \mathrm{P}^{+}\right)=\overline{\mathrm{C}}^{\infty}\left(\mathrm{BPh}_{4}^{-}\right)=1141 \pm 1 \mathrm{~J} \mathrm{~K}^{-1} \mathrm{~mol}^{-1}$. The TATB assumption has then been employed by Abraham and Marcus (ref. 7) together with the additivity rule to obtain the values of the infinite dilution partial molar heat capacities of other ions. The standard molar heat capacities of gaseous monoatomic ions are simply (5/2) R, but those of polyatomic ions must be obtained from structural and spectroscopic data. They have recently been calculated by Marcus and Loewenschuss for over 130 such ions for the temperature range 100 to $1000 \mathrm{~K}$ and presented in tables (ref. $3)$. The values calculated for $298.15 \mathrm{~K}$ in conjunction with the $\bar{C}_{p}{ }^{\infty}$ (aq) values yield the heat capacities of hydration of many ions.

The present model is similar in many respects to that employed by Abraham and Marcus (ref. 7), except that it utilized a variable value of $\Delta r$, rather than the fixed value of $0.15 \mathrm{~nm}$ (approximately the radius of a water molecule) used previously. The neutral term is $\Delta C_{\text {pNt }}=-55+1380(\mathrm{r} / \mathrm{nm})$ and the electrostatic ones are $\Delta C_{P E \|}+\Delta C_{P E / 2}=-2.07 \mathrm{z}^{2}[7.83(\Delta \mathrm{r} / \mathrm{r})+2.83] /(\mathrm{r}+\Delta \mathrm{r})$ $\mathrm{J} \mathrm{K}^{-1} \mathrm{~mol}^{-1}$, as in ref. 7. The resulting values of the structural effects term are shown in Table 1. They indicate the water-structure effects of the ions in almost the same way as do the corresponding entropy values also shown in this table. The zero point for $\Delta C_{p S t}$ was taken as $-140 \mathrm{~J} \mathrm{~K}^{-1}$ mol for ions that have little structural effects (tetraethylammonium and potassium, rather than sodium); water-structure-breaking ions have more negative values of $\Delta C_{p S t}$, water-structure-enhancing ones have less negative or even positive values.

\section{STANDARD PARTIAL MOLAR VOLUME OF AQUEOUS IONS AT 298.15 K}

The standard molar volume of all ions in the (ideal) gas phase is the same, $\mathrm{RT} / \mathrm{P}_{0}$. Hence their standard molar volume of hydration does not convey more information concerning the interactions on hydration than does their partial molar volume in aqueous solution at infinite dilution. On the contrary, the quantity $\Delta_{\text {hyd }} \mathrm{V}^{\circ}$ obscures the individual ionic properties, since it has the same magnitude as $\mathrm{RT} / \mathrm{P}_{0}$ and is more than two orders of magnitude larger than $\bar{V}^{\infty}(\mathrm{aq})$. The volume of hydration, $\Delta_{\text {hyd }} \mathrm{V}^{*}$ according to eq. (5), does not suffer from this difficulty, but hardly adds more information than does $\nabla^{\infty}(a q)$. 
The splitting of the partial molar volumes of aqueous electrolytes into individual ionic contributions by means of the TATB assumption can be made according to Millero (ref. 22, 23). He took into account the small discrepancy in sizes of its constituent ions. Depending on how this was done, a value of $13.2 \pm 0.9 \mathrm{~cm}^{3} \mathrm{~mol}^{-1}$ was obtained for the difference in $\bar{v}^{\infty}$ of tetraphenylarsonium and tetraphenylborate, leadind to the average partial molar volume at infinite dilution of $295.7 \mathrm{~cm}^{3}$ mol-1 for $\mathrm{Ph}_{4} \mathrm{As}^{+}$and $282.6 \mathrm{~cm}^{3} \mathrm{~mol}^{-1}$ for $\mathrm{BPh}_{4}$. These values for the reference ions lead to $\overline{\mathrm{V}}^{\mathrm{D}}=-5.0 \mathrm{~cm}^{3} \mathrm{~mol}^{-1}$ for $\mathrm{H}^{+}$, in good accord with experimental values, obtained by Zana and Yeager (ref. 24) from vibration potential measurements. simple halving of the partial molar volume of TATB would lead to $\overline{\mathrm{v}}^{\infty}\left(\mathrm{H}^{+}, \mathrm{aq}\right)=-11.6 \mathrm{~cm}^{3} \mathrm{~mol}^{-1}$, which is much more negative than other reasonable values that have been suggested, see Conway (ref. 17) and Millero (ref. 23). The values of $\bar{\nabla}^{\infty}(a q)$ of many ions at $298.15 \mathrm{~K}$ are listed in the latter work.

Application of eqs. (5) and (10) tests the model on these data. The right hand side of eq. (5) differs from $\overline{\mathrm{V}}(\mathrm{aq})$ by only RTK $=1.1 \mathrm{~cm}^{3} \mathrm{~mol}^{-1}$ at 298.15 K. The neutral term was taken in this case as the intrinsic volume (ref. 23), $\Delta V_{N t}=4 \pi N_{A v} r^{3} / 3=2520(\mathrm{r} / \mathrm{nm})^{3}$. The electrostatic terms, arising from the pressure derivatives of $\varepsilon^{\prime}$ and $\varepsilon$, are $\Delta V_{E \|}+\Delta V_{E / 2}=-1.25 z^{2} \times$ $[(\Delta r / r)+0.05] /(r+\Delta r)$, only a very minor portion of the electrostriction being ascribable to the region beyond $r+\Delta r$ from the center of the ion.

The structural effects on the volume, $\Delta V_{S t}$, obtained as for the other functions by difference, are shown in Table 1. They are not directly related to the negative $\Delta S_{S t}$ and the positive $\Delta C_{p S t}+140 \mathrm{~J} \mathrm{~K}-1$ mol-1 for water structure-making ions (and vice versa for water-structure-breaking ones). still, the positive $\Delta v_{S t}$ for multivalent cations is indicative of the additional volume created by the enhanced hydrogen bonding near the ions caused by these structure-making ions. On the other hand, no decrease in volume is shown by the structure-breaking large hydrophilic univalent ions. The hydrophobic tetraalkylammonium ions, even the smaller ones like tetramethylammonium, do produce considerable additional volume on hydration.

\section{REFERENCES}

1. A. Ben-Naim and Y. Marcus, J. Chem. Phys., 81, 2016-2027 (1984).

2. A. Loewenschuss and Y. Marcus, Chem. Rev., $\overline{84}, 89-115$ (1984).

3. A. Loewenschuss and Y. Marcus, J. Chem Phys. Ref. Data, 16, in the press (1987).

4. Y. Marcus, Pure Appl. Chem., 55, 977-1021 (1983).

5. Y. Marcus, Pure Appl. Chem., 57, 1103-1128 (1985).

6. Y. Marcus, Pure App1. Chem., 58, 1721-1736 (1986).

7. M.H. Abraham and Y. Marcus, J. Chem. Soc., Faraday Trans. 1, 82, 3255$3267(1986)$.

8. Y. Marcus, J. Chem. Soc., Faraday Trans. 1, submitted (1986).

9. M.H. Abraham and J. Liszi, J. Chem. Soc., Faraday Trans. 1, 74, 1604$1614,2858-2867$ (1978).

10. A.I. Rusanov and F.M. Kuni, Zh. Fiz. Khim., 58, 2336-2338 (1984); M. Bucher and T.L. Porter, J. Phys. Chem., 90, 3406-3411 (1986).

11. Y. Marcus, J. Soln. Chem-, $\overline{12,} 27 \overline{1-275}(\overline{1983})$.

12. Y. Marcus, J. Chem. Soc., Faraday Trans. 1, 82, 233-240 (1986).

13. M.H. Abraham, E. Matteoli, and J. Liszi, J. Chem. Soc., Faraday Trans. 1, 79, 2781-2800 (1983); M.H. Abraham and J. Liszi, ibid., 76, 1219-1231 $(19 \overline{80})$.

14. W.M. Latimer, K.S. Pitzer, and C.L. Slansky, J. Chem. Phys., 7, 108-11 (1939) .

15. D.D. Wagman, W.H. Evans, V.B. Parker, R.H. Schumm, I. Halow, S.M. Bailey, K.L. Churney, and R.L. Nuttall, J. Phys. Chem. Ref. Data, 11, Suppl. 2 (1982).24.

16. Y. Marcus, J. Chem. Soc., Faraday Trans. 1, 83, in the press (1987).

17. B.E. Conway, J. Soln. Chem.' 7, 721-770 (1978).

18. Y. Marcus and A. Loewenschuś, J. Chem. Soc., Faraday Trans. 1, 82, 993 (1986).

19. T. Davies and L.A.K. Stavely, Trans. Faraday Soc., 53, 19-30 (1957).

20. B.G. Cox and A.J. Parker, J. Am. Chem. Soc., 95, 6879-6884 (1973).

21. C. Jolicoeur, P.R. Philip, G. Perron, P.A. Leduc, and J.E. Desnoyers, Can. J. Chem., 50, 3167-3178 (1972) .

22. F.J. Millero, J. Phys. Chem., 75, 280-284 (1971).

23. F.J. Millero, Chem. Rev.'71, 147-176 (1971).

24. R. Zana and E. Yeager, J. Phys. Chem., 71, $521-536$ (1967). 